\title{
Economic Evaluation of Mammography-Based Breast Cancer Screening Program in Saudi Arabia
}

\author{
Awadh Alqahtani, MD, FRCSC \\ Bariatric Surgery and Surgical Oncology Consultant, King Saud University, Saudi Arabia
}

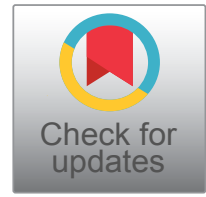

*Corresponding author: Awadh Alqahtani, MD, FRCSC, Bariatric Surgery and Surgical Oncology Consultant, King Saud University, Riyadh, Saudi Arabia, Tel: 00966552894444

\begin{abstract}
Introduction: Breast cancer is the commonest malignancy among Saudi women, accounting for $23.6 \%$ of all female cancers. It has been the most frequent cancer in Saudi women for the last 30 years.

Objectives: To determine the cost effectiveness of a breast cancer screening program based on mammography in Saudi Arabia versus the alternative option of treating only those cases already symptomatic or incidentally found (i.e. the current practice).

Material and methods: We used a probabilistic model to estimate the effect and costs over time of each scenario. The effectiveness of mammography screening measured as years of life (YL), quality-adjusted life years (QALY), Costs of screening and treatment obtained from the hospital databases in Saudi Arabia.

The economic analysis of mammography screening carried out as a cost-effectiveness analysis. A decision tree based on a Markov model used in estimating effectiveness and costs. The purpose of the model is to integrate the following parameters: Screening costs, diagnostic costs, health care costs, discount rate and potential effectiveness of the evaluated program, measured by deaths avoided or years of life saved. The incremental cost-effectiveness ratio (ICER) used to compare the relative costs and outcomes of different scenarios. All costs presented in 2014 Saudi riyals. Costs in other currencies will be converting at the average international exchange rate for April 2014. Costs from other periods in time adjusted using the Saudi Health Care Price Index.
\end{abstract}

Results: The cost-effectiveness ratio per avoided death is 577,500 SAR and per saved life year 36,500 SAR. The sensitivity analysis showed the efficacy of mammography, compliance of the program and screening costs to be the more sensitive variables.
Conclusion: The mammography screening program strategy generates, in all analysis scenarios, an additional cost per avoided death as well as per life year saved when compared to the no-screening alternative.

\section{Keywords}

Mammography, Breast cancer, Breast screening

\section{Introduction}

The breast cancer (BC) is the most common malignancy in the women worldwide. Approximately 1.7 million new cases diagnosed in 2012, which represent a quarter of all cancer in the women. The Belgium is the highest rate of breast cancer incidence-111.9 age standardized rate per 100,000 -followed by Denmark and France [1].

Although the breast cancer one of the commonest cancer in Asia, but the incidence rate remains low. The highest incidence rate of $\mathrm{BC}$ in Asia in Pakistan (50 per $100,000)$ followed by Singapore and Philippines. These numbers are low when we compared with Western countries, but the incidence is increasing more rapidly in Asia than the west due to the change in women life styles and diet which be similar to the western countries [2].

In the Gulf Cooperation Council (GCC) countries, there are 4480 females diagnosed with breast cancer during 1998-2002. The age-standardized incidence rate per 100,000 was ranged highest in Bahrain (46.4), followed by Kuwait (44.3), Qatar (35.5), United Arab Emirates (19.2), Oman (14.4) and Saudi Arabia (12.9) [3]. Saudi National Cancer Registry in 2010 reported the

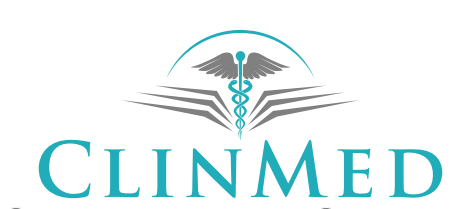

INTERNATIONAL LIBRARY
Citation: Alqahtani A (2020) Economic Evaluation of Mammography-Based Breast Cancer Screening Program in Saudi Arabia. Int J Surg Res Pract 7:119. doi.org/10.23937/2378-3397/1410119

Accepted: October 31, 2020; Published: November 02, 2020

Copyright: (C) 2020 Alqahtani A. This is an open-access article distributed under the terms of the Creative Commons Attribution License, which permits unrestricted use, distribution, and reproduction in any medium, provided the original author and source are credited. 
fist common malignancy in the women is breast cancer, which represents $25 \%$ of newly diagnosed female cancers, and the ASR was 19.2/100,000 for female population [4].

All these values are leading us to search about the benefits of establishing the national breast cancer-screening program from the economic view. In general the economic evaluation is an aid to decision making, and help us to reach a value judgment as to whether or not to provide a particular screening program $[5,6]$. One of the economic evaluations is Economic cost effective study, which evaluates a health intervention by asking, how much health benefit do we get for our money? $[7,8]$.

The aim of this study is to perform economic analysis of mammography screening carried out as a cost-effectiveness analysis, versus the alternative option of treating only those cases already symptomatic or incidentally found (i.e. the current practice) in Saudi Arabia 2014.

\section{Material and Methods}

\section{The model and the parameters}

A decision tree based on a Markov model was used in estimating effectiveness and costs. The purpose of the model is to integrate the following parameters: Screening costs, diagnostic costs, mammography sensitivity and specificity, health care costs, discount rate and potential effectiveness of the evaluated program, measured by deaths avoided or years of life saved. In the model the disease occurred according to predefined probabilities. We used a discount rate of $3 \%$ in the breast cancer screening Mammogram, Gold, et al. Because given currently available economic data, the U.S. Public Health Service recommends 3\% as the most appropriate real risk less discount rate for economic analyses by the Panel on Cost-effectiveness in Health and Medicine appointed in 1993 [9].

To perform the cost-effectiveness analysis a decision tree was designed (Figure 1) where 2 alternative options have been compared:

1. No screening program, which is the current practice in most parts of Saudi Arabia.

2. A mammography-screening program through personal appointments, with most women between 40 and 65-years-old.

The effectiveness of mammography screening is measured as years of life (YL), quality-adjusted life years (QALY), Costs of screening and treatment were obtained from the hospital databases in Riyadh city, Saudi Arabia. The economic analysis of mammography screening was carried out as a cost-effectiveness analysis.

The evidence for the effectiveness of Mammogram derived from a review of previously completed studies

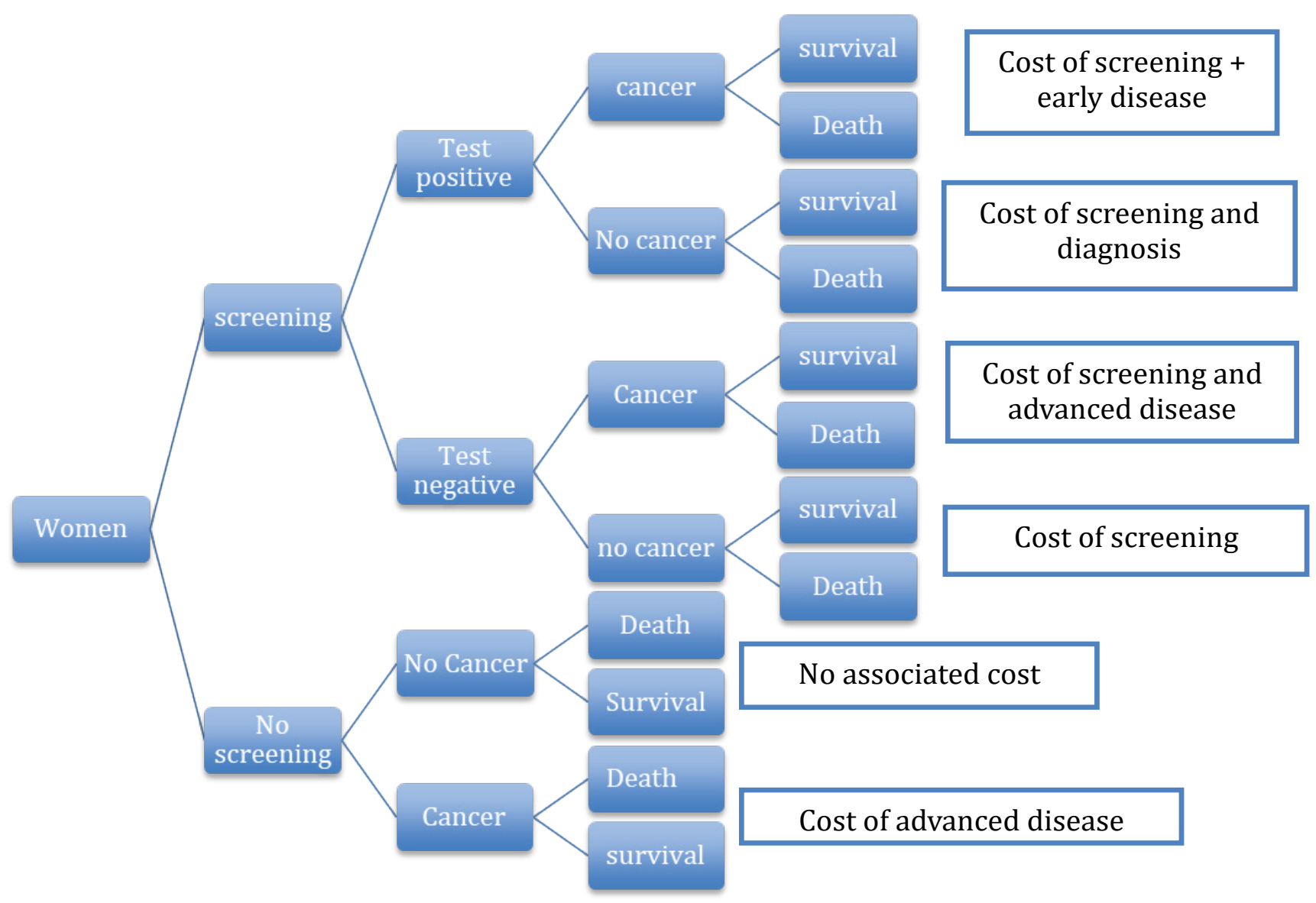

Figure 1: To perform the cost-effectiveness analysis a decision tree was designed. 
and the efficacy of mammography was taken from studies considering women of similar age and with similar time intervals, also we assessed the Criteria and the Methods that used to judge relevance and validity of primary studies, after we included the primary studies, we did the critical appraisal for each study. And then we did our mammogram effectiveness analysis to assess the following: Sensitivity and specificity of mammography, mammogram effectiveness (as measured by the percentage of breast cancer mortality reduction) and screening program compliance, which was set as 0.90 and $0.98,30 \%, 0.70$ respectively [10].

\section{Perspectives}

We evaluated the costs from the societal perspective, which includes health-care costs, non-health Care costs.

\section{Cost data and resources}

The incremental cost-effectiveness ratio (ICER) was used to compare the relative costs and outcomes of different scenarios. Lives year saved (LYS) and the cost per quality-adjusted life year saved (QALY) were used as outcome measures in the Cost-effectiveness analysis. Outcomes of interest include number of and cost per cancer detected by screening breast cancer deaths prevented, life -years gained, and quality adjusted life -years gained $[11,12]$.

The data for the mammogram effectiveness analysis and the use of resources will be collect in 2014. Projections of data will be till 2034 based on age-adjusted mortality rates. 2014 prices have been used $[8,9,11,12]$.

All costs presented in 2014 Saudi riyals. Costs in other currencies were converting at the average international exchange rate for July 2014. Costs from other periods in time were adjusted using the Saudi Health Care Price Index.

The costs divided according to Gold, et al. into health care, non-health care and time costs. Health care costs included also the health care savings caused by the screening program. Non-health care costs are travel costs. Costs calculation Program includes both direct costs to the health system and indirect Costs generate by screened women (transportation, loss of working hours, mammography-associated intangible costs) [12]. In our study, we focused only on the direct cost which is the health system cost included surgery costs, follow-up costs and associated therapy costs (chemotherapy, radiation therapy and hormone therapy). Contrary, we could not get the indirect cost because of lack of data.

The costs divided into fixed (independent of the number of screened women) and variable (depending on that number). Fixed costs include human resources, advertising, information and amortizations. Variable costs include repairing and maintenance of mammographic equipment, X-ray film, traveling expenses, stationery, training activities, other activities and other expenses (Table 1) [11-13].

\section{Cost effective economic analysis}

Cost-effectiveness analyses evaluate a health intervention by asking, how much health benefit do we get for our money? The reason why we chose this analyses is to help decision makers allocate health care resources efficiently $[7,8]$. CEA express costs in monetary units and health benefits in units of health, such as a life saved, a case of cancer averted, or a quality-adjusted life-year (QALY) gained $[11,14,15]$. The analyses compare breast cancer (BC) mammography screening program, with no program alternative. So we calculated the cost-effectiveness as the difference in cost between mammogram screening and no screening, divided by the difference in health benefit obtained with screening and without screening. The cost effectiveness ratio calculation was performing in a computer-spread sheet. It allowed the integration of the following parameters: Screening costs, diagnostic costs, mammography sensitivity and specificity, health care costs, discount rate, and potential effectiveness of the evaluated program measured by avoided deaths or saved years of life. Future costs and benefits have been updated at a $3 \%$ discount rate $[11,12]$.

\section{Sensitivity analysis}

A sensitivity analysis conducted in those cases in

Table 1: Variable costs include repairing and maintenance of mammographic equipment, $X$-ray film, traveling expenses, stationery, training activities, other activities and other expenses.

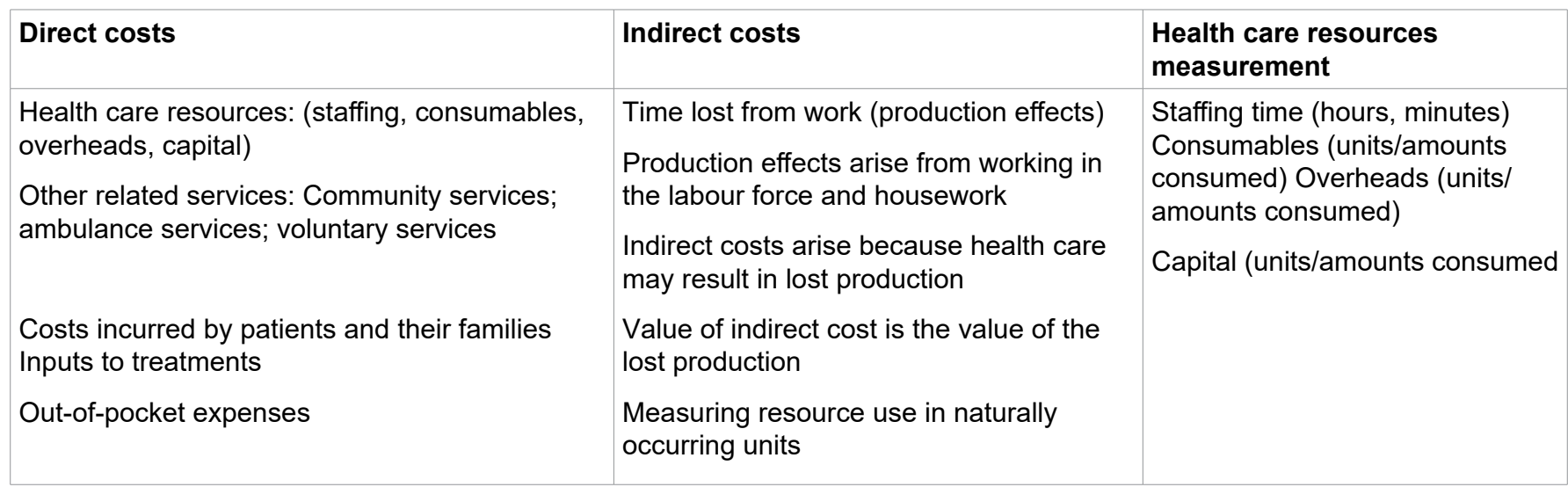


which there is uncertainty regarding the validity of data. The following variables investigated: Highest incidence and mortality associated with breast cancer, program compliance, specificity, highest and lowest efficacy (several values depending on age groups), and a discount rate, Uncertainty related to variables that carry value judgment was subjected to one-way sensitivity analysis using a $3 \%$ discount rate for both costs and health effects, with age weighting. Also we examined the sensitivity of the results to the use of a $0 \%$ discount rate for health effects, $3 \%$ for costs, and with no age-weighting $[12,16]$.

\section{Results}

(We should add how many patients, The incremental cost-effectiveness ratio (ICER), quality-adjusted life year

Table 2: Diagnostic and treatment costs.

\begin{tabular}{|l|l|l|}
\hline Women & $\begin{array}{l}\text { Treatment } \\
\text { cost }\end{array}$ & $\begin{array}{l}\text { Diagnostic } \\
\text { cost }\end{array}$ \\
\hline Non screened woman & 17500 SR & 3500 SR \\
\hline \multirow{2}{*}{ Screened } & $(4730 \$)$ & $(946 \$)$ \\
\hline & 13500 SA & 2100 SA \\
\hline & $(3649 \$)$ & $(568 \$)$ \\
\hline
\end{tabular}

saved (QALY), Sensitivity analysis).

After calculating the needed costs, which are program costs, Diagnostic associated costs, and Health care costs, we ended up with these cost assumptions that the treatment cost and the diagnostic cost being higher in non-screened women (21000 SR) than screened women (15600 SR) (Table 2).

The program costs only include the direct costs to the health system, and the average cost per screened woman was set at 350 SR. Moreover, the diagnostic associated costs have three categories, which is breast cancer diagnosed through mammogram, interval of breast cancer and false positive breast cancer (Table 3). Health care costs composed of treatment costs and follow up costs in Markov Models.

The results showed that the cost of the screening programs during the upcoming 25 years would be $2,813,823$ SR compared to without the screening which would be $1,225,300 \mathrm{SR}$. In addition, the death due to breast cancer has decreased with 1,118,755 life years saved. The cost effectiveness of the screening programs was 577,500 per avoided death, and 36,500 per saved life years (Table 4).

Table 3: The diagnostic associated costs have three categories, which is breast cancer diagnosed through mammogram, interval of breast cancer and false positive breast cancer.

\begin{tabular}{|l|l|l|}
\hline Symptoms & Procedure & Cost \\
\hline Unilateral discharge & Medical care, mammogram & 650 SR (176\$) \\
\hline Bilateral discharge & Medical care, mammogram labs & 1100 SR $(297 \$)$ \\
\hline Non palpable mass & Medical care, mammogram labs, localizing X-ray, Bx & 1200 SR (324\$) \\
\hline Palpable mass/staging & Bx, pathology, labs, radiology & 4000 SA (1081\$) \\
\hline Screening & Bx, pathology, labs, radiology & 2100 SA (568\$) \\
\hline False positive & Biopsy & 550 SA (149\$) \\
\hline
\end{tabular}

Table 4: The cost effectiveness of the screening programs was 577,500 per avoided death, and 36,500 per saved life years.

\begin{tabular}{|l|l|l|}
\hline \multicolumn{2}{|l|}{ With screening } & Without screening \\
\hline Cost & $2,520,105$ SR $(681,110 \$)$ & \\
\hline 1-Screening & $1,79,915$ & $2,12,580$ \\
\hline 2-Diagnosis & $(48,626 \$)$ & $(57,454 \$)$ \\
\hline 3-Treatment and follow up & $1,13,803$ & $10,12,720$ \\
\hline Total & $(30,758 \$)$ & $(273,708 \$)$ \\
\hline Effects & $28,13,823$ & $(3,25,300$ \\
\hline 1-Death due to breast cancer & $(760,493 \$)$ & \\
\hline 2-Life years saved & & $5,29,050$ \\
\hline Cost-effectiveness & $4,52,450$ & \\
\hline Per avoided death & $11,18,755$ & \\
\hline Per saved life years & & \\
\hline
\end{tabular}




\section{Discussion}

In the Kingdom of Saudi Arabia, breast cancer mostly diagnosed unfortunately in its late stages, and the reason goes back to the presentation, where the patients come to the hospitals already symptomatic. Therefore, the prognosis for those diagnosed patients sadly turns to be poor. And here where the importance of breast cancer screening comes, to detect cancer as early as possible and subsequently having a better prognosis. So, our study tries to test the cost-effectiveness of breast cancer screening programs by mammography and estimates its long-term benefits in order to implement it.

A Sarvazyan, et al. discussed the cost effectiveness of breast cancer screening programs worldwide. They presented the results in the costs of mammography screening being relatively compared to the income of physicians. It shows that the low income (developing countries such as India, Egypt and Iran) almost reached $1,000 \%$ of physician income, while the high income (developed countries such as US, UK and Australia) they didn't reach $100 \%$ of physician income [17].

After collecting the data and calculating the costs by using the cost-effectiveness analysis, the estimated benefits from the screening programs are 13,320 saved lives and 223,751 gained life years, which support the importance of breast cancer screening programs. But as mentioned in the results, the costs with the screening $(2,813,823)$ seem to be double the costs without the screening $(1,225,300)$. Unfortunately, this high difference leaves us to the end of are study being not cost-effective.

E. Barfar, conducted a similar study in Iran, in ten cities. The study included more than 26,000 women with the age 35 and above, and selected low socioeconomic background. They estimated a total cost of breast cancer careening program of $\$ 377,797$, and identified 24 breast cancer patients. Unfortunately the number is close to the baseline incidence without the screening, upon that, the study concluded that breast cancer screening programs are not cost effective to be implemented in Iran. The study reported a few technical efficiency problems contributed to the end result [18].

In comparison with our study, the results are similar, which tells us that breast cancer screening programs are not cost-effective in the developing countries therefore it doesn't support their implementation. In our study we found many reasons that led to the end results of our research not being cost effective. The assumed period of the research is believed to be too long and contributing to the misled results. Another factor is that the incidence of breast cancer in Saudi Arabia is very low due to the inaccuracy of the registration, and that also caused the wrong difference between the cost with the screening and without the screening.

In the future, further advanced researches need to be conducted with the accurate incidence of breast can- cer in Saudi Arabia in order to proof that breast cancer screening programs are cost-effective, so they can be applied in the country and help in reducing the mortality rates and in return increasing the survival rates. So, previous researches for the incidence of breast cancer in Saudi Arabia need to be conducted first.

\section{Conclusion}

To conclude, our study results show additional costs per avoided death and per life year saved with the use of mammography screening programs. Along with multiple influencing factors, these additional costs led our study to be not cost-effective.

\section{References}

1. Breast cancer statistics.

2. Verma M (2009) Cancer epidemiology, New York.

3. Ravichandran K, Al-Zahrani A (2009) Association of reproductive factors with the incidence of breast cancer in Gulf Cooperation Council countries. East Mediterr Health J 15: 612-621.

4. Saudi Cancer Registry.

5. Khadiga D, Al-Mohaimeed A (2007) Knowledge, attitudes and practices surrounding breast cancer and screening in female teachers of Buraidah, Saudi Arabia. Int J Health Sci 1: 76-85.

6. Ceber E, Turk M, Ciceklioglu M (2010) The effects of an educational program on knowledge of breast cancer, early detection practices and health beliefs of nurses and midwives. J Clin Nurs 19: 2363-2371.

7. Guidelines for the early detection and screening of breast cancer. WHO.

8. Brown DW, French MT, Schweitzer ME, McGeary KA, McCoy CB, et al. (1999) Economic evaluation of breast cancer screening: A review. Cancer Pract 7: 28-33.

9. Brown ML (1992) Economic considerations in breast cancer screening of older women. J Gerontol 47: 51-58.

10. Elwood JM, Cox B, Richardson AK (1993) The effectiveness of breast cancer screening by mammography in younger women. Online J Curr Clin Trials.

11. Detsky AS, Nagle IG (1990) A clinician's guide to cost effectiveness analysis. Ann Intern Med 113: 147-154.

12. Drummond M, O'Brien B, Stoddart G, Torrance G (1997) Methods of economic evaluation of health care programmes. ( $2^{\text {nd }}$ edn), Oxford University Press.

13. Koning De HJ (2000) Breast cancer screening: Cost-effective in practice? Eur J Radiol 33: 32-37.

14. Gold MR, Siegel JE, Russell LB, Weinstein MC (1996) Cost-effectiveness in health and medicine. Oxford University Press, New York.

15. Garber AM, Phelps CE (1997) Economic foundations of cost-effectiveness analysis. J Health Econ 16: 1-31.

16. Briggs A, Sculpher M (1995) Sensitivity analysis in economic evaluation: A review of published studies. Health Econ 4: 355-371.

17. Sarvazyan A, Egorov V, Son JS, Kaufman CS (2008) Cost-effective screening for breast cancer worldwide: Current state and future directions. Breast Cancer 1: 91-99.

18. Barfar E, Rashidian A, Hosseini H, Nosratnejad S, Barooti $\mathrm{E}$, et al. (2014) Cost-effectiveness of mammography screening for breast cancer in a low socioeconomic group of Iranian women. Arch Iran Med 17: 241-245. 